\title{
Investigation of the Usage of Oil Palm Harvesting Tools on Work Related Musculoskeletal Disorders of Lower Limb
}

\author{
Shahrol Mohamaddan ${ }^{1,2^{*}}$, Magdalene Andrew-Munot ${ }^{2}$, Shirley Johnathan Tanjong ${ }^{2}$, \\ Siti Zawiah Md Dawal ${ }^{3}$, Keith Case $^{4}$ and Akihiko Hanafusa ${ }^{1}$
}

\begin{abstract}
${ }^{1}$ Department of Bioscience and Engineering, College of Systems Engineering and Science, Shibaura Institute of Technology, 337-8570 Saitama, Japan

${ }^{2}$ Department of Mechanical and Manufacturing Engineering, Faculty of Engineering, Universiti Malaysia Sarawak, 94300 Kota Samarahan, Sarawak, Malaysia

${ }^{3}$ Department of Mechanical Engineering, Faculty of Engineering, University of Malaya, 50603 Kuala Lumpur, Malaysia ${ }^{4}$ Mechanical, Electrical and Manufacturing Engineering, Loughborough University, Leicestershire, LE11 3TU, United Kingdom

*Corresponding Author: Shahrol Mohamaddan, Email: mshahrol@shibaura-it.ac.jp
\end{abstract}

\begin{abstract}
Worker's health and safety are important for the plantation's sustainable development. In the oil palm industry, the harvester is exposed to work-related musculoskeletal disorders (WMSDs) during the fresh fruit bunch (FFB) harvesting process. The objective of this study was to investigate the effect of harvesting tools namely chisel, pole, and loading spike towards WMSDs on harvester's lower limb. Rapid Upper Limb Analysis (RULA) in the CATIA and Human Musculoskeletal Model Analysis (HMMA) in the AnyBody software was applied to model and simulate the harvester's movement during the harvesting process. The RULA simulation results indicated that the harvester's lower limbs were exposed to WMSDs where further investigation and changes were required immediately. HMMA results indicated that the Gastrocnemius Medialis muscle had the highest mean muscle force. This study is important as an early ergonomics intervention for occupational health.
\end{abstract}

Keywords: oil palm, manual harvesting, sustainable development, RULA, HMMA

\section{Introduction}

The oil palm industry is the largest contributor to the global production of oils and fats. Malaysia is one of the biggest producers with five million hectares of oil palm is under cultivation [1]. In oil palm plantations, the harvesting process is crucial for producing high-quality oil [2]. The harvesting process needs to be conducted when the fruit is at optimal ripeness to obtain a high oil content level [3]. In total, the oil palm remains productive up to the age of 32 years [4]. However, harvesting oil palm fresh fruit bunches (FFB) is a laborious and hazardous task. In general, harvesters use three types of tools namely the chisel, pole, and loading spike for the harvesting process.

Figure 1 shows the oil palm FFB harvesting by using a pole. The pole has a rounded design and a sickle cutter is attached at the end of it. The pole is usually used for trees above 3 meters in height. When using a pole to cut the FFB, the harvester is required to pull and push the pole strongly. In general, several pulling and pushing motions are needed in each session depending on the harvester's skill. Both hands are normally above shoulder level and the body is leaning forward to perform the task. For trees lower than 3 meters, the chisel is usually used. The chisel is a rounded design tool with a trapezium cutter shape at the end of it. When using a chisel, the harvester needs to hold and swing the tool strongly. Normally, the knees are bending, and the body is leaning forward to cut the FFB using a chisel. The loading spike is used to lift and collect the FFB into the wheelbarrow. The loading spike is also rounded with a sharp edge at the end. When using a loading spike, the harvester's body is bending forward to lift the bunch of FFB from the ground. Different body postures, repetitive work, and prolonged standing during harvesting the oil palm FFB may contribute to muscle fatigue [5].

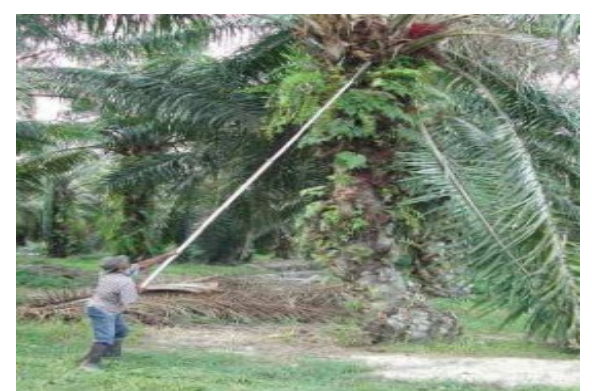

Fig. 1. Oil palm harvesting using the pole [6]

Muscle fatigue was identified as a major factor that leads to work-related musculoskeletal disorders (WMSDs) [7]. WMSD is the condition in which the harvester undergoes fatigue and discomfort at their body parts where swelling, pain in the joints, and tingling happen [8]. It causes underlying damage to the tissue thus causing pain and disability of muscles [9]. Repetitive work is defined as an unvaried job with an extreme level of frequency in each period. According to Ref. [10], repetitions are conditional based on the work cycle. High repetitions happen when the worker motion cycle time is less than 30 seconds of the average cycle time [11]. According to Ref. [12], when a task is done repeatedly for more than $60 \mathrm{~min}$, it could also be considered repetitive work. Prolonged standing can cause muscle-related disorders that can lead to lower back pain [13]. Based on research conducted by Ref. [14], 50\% of healthy respondents reported discomfort in the lower back when exposed to more than 2 hours of continuous standing.

Although many automated machines have been designed and developed [15], manual tools are still widely used to harvest the oil palm FFB due to cost-effectiveness. The objective of this study was to investigate the usage of different harvesting tools towards WMSDs on harvester's lower limbs. 